\title{
Modified Atkins Diet for Tics Requiring Treatment in Tourette Syndrome: A Randomized Controlled Trial of Early Versus Late Initiation
}

Camilla B Sørensen, $M D^{1}$, Liselotte Skov, MD, MSc ${ }^{1}$, Lone Aaslet, $N P^{1}$, Helle Nielsen, $R D^{2}$, Mette Mortensen, $R D^{3}$, Theis Lange, $P h D^{4}$, Nanette Mol Debes, $M D, P h D^{1}$ and Maria J Miranda, MD, $P h D^{1^{*}}$

${ }^{1}$ Department of Pediatrics, Herlev University Hospital, Denmark

${ }^{2}$ Filadelfia Epilepsy Hospital, Denmark

${ }^{3}$ Rigshospitalet, Copenhagen, Denmark

${ }^{4}$ Section of Biostatistics, University of Copenhagen, Denmark

\begin{abstract}
Increased dopamine activity is thought to play a role in the pathophysiology of Tourette syndrome (TS). Since ketogenic diet $(K D)$ is thought to change several neurotransmitter systems, we hypothesized that modified KD could be beneficial in treatment of tics.

A single-site prospective study with intention-to-treat design was performed with 26 children with TS. After an observation period, participants were randomized into two groups: Group a started diet immediately, and group B started after a further observation period of 3-5 months. Participants were examined with validated diagnostic instruments to assess severity of tics and comorbidity at diet start, after one month and thereafter every three months. The mean number of days on diet was 65.62 days. The diet was well tolerated but difficult to maintain. There were no significant differences in effect of diet between the groups or over time, but a tendency towards positive effect of diet was seen $(p=0.1025)$.
\end{abstract}

\section{Keywords}

Tourette syndrome, Ketogenic diet, Non-pharmacological treatment

\section{Introduction}

Tourette syndrome (TS) is a chronic neurobiological movement disorder characterized by the presence of multiple motor and vocal tics persistent for more than one year [1]. Tic onset is usually around the age of 4-6-years $[2,3]$ and tics usually have a waxing and waning course. The severity of tics declines during adolescence, but more than $80 \%$ of adolescents over 16 -years still have tics [4]. TS is often associated with comorbidities. The most frequent comorbidities are attention deficit hyperactivity disorder (ADHD) and obsessive-compulsive disorder (OCD) [5].

The complex pathogenesis of TS is not completely understood, but increased dopamine activity is thought to play a role in the development of tics $[6,7]$.

When to treat tics depends on subjective impairment of the patient. Some TS patients with objective severe tics only experience mild impairment, whereas others with only mild tics experience significant suffering [8]. European treatment guidelines suggest that pain or injury, social or emotional problems and/or functional interference caused by tics should lead to treatment [9].

There are several treatment possibilities for tics, including pharmacological treatment and behavioral therapy $[9,10]$. Historically, pharmacological treatment has been the most commonly used strategy in the treatment of tic disorders, but the latest years treatment choice has changed towards non-pharmacological treatment as primary option partly to avoid a wide range of advers effects [11-13].

Today European guidelines recommend behavioral thera-

*Corresponding author: Maria J Miranda, MD, PhD, Department of Pediatrics, Herlev University Hospital, Borgmester, Ib Juuls Vej 25C, $3^{\text {rd }}$ Floor 2730 Herlev, Denmark, Tel: +45-38-6810-87

Accepted: March 16, 2021

Published online: March 18, 2021

Citation: Sørensen CB, Skov L, Aaslet L, et al. (2021) Modified Atkins Diet for Tics Requiring Treatment in Tourette Syndrome: A Randomized Controlled Trial of Early Versus Late Initiation. J Pediatr Neurol Neurosci 5(1):86-94 
py $[14,15]$ as first-line interventions for tics for children and adolescents who experience mild-to-moderate tic severity $[10,16]$. While behavioral therapies have demonstrated their efficacy for managing tics, there are still some limitations using these in a clinical setting, among others a very limited number of clinicians trained in administering the treatment. Comorbidities and age can make this difficult, why alternative treatment options should be available.

The ketogenic diets (KDs) - either classical or modified forms - are high fat, low carbohydrate and adequate protein diets used for treatment of severe medicine-intractable epilepsy [17]. The evidence in epilepsy is huge [17], where at least $50 \%$ of children with medicine-resistant epilepsy will achieve at least $50 \%$ reduction and even seizure freedom on the KD. However, there is increasing evidence that the KDs are potential treatments for many other neurological diseases $[18,19]$ as the mechanisms of action of the KDs are various and diverse [20-22]. Although we do not understand all the mechanisms yet, there is already plenty of evidence on several of these mechanisms [22]. We know that the KD influences several systems in the brain that in the end will increase the mitochondrial energy capacity and stabilize the membrane of the neurons [21,22]. Other potential mechanisms of action are effects on the mammalian target of rapamycin (mTOR) pathway, increase of GABA [20-23] activity or modulation in Dopamine system [24].

With this in mind and knowing that imbalanced dopamine activity is probably a pathogenic mechanism in tics, we hypothesized that a modified KD (Modified Atkins Diet $=$ MAD) could be beneficial for children and adolescents with tics requiring treatment in reducing tics frequency and severity with fewer adverse effects than pharmacological therapy.

\section{Methods and Study Design}

\section{Design}

Single-site study conducted at the national Danish To- urette Clinic at Herlev University Hospital, department for children and adolescents, Denmark, with an inclusion period between January 2014 and December 2015. The clinic is a centralized national Tourette clinic, that performs evaluation, treatment, follow up and research concerning children with TS and tic-related diseases. This study was a prospective study with intention-to-treat design. Written informed consent was obtained from all participants.

After obtained consent, all participants underwent an observation period of 2 months (Figure 1). Thereafter participants were blindly randomized into two groups: Group A that started diet immediately after randomization, and group $B$, who underwent a further observation period of 3-5 months, starting diet after this period. The design was chosen in order to minimize the influence of the natural fluctuating course of tics in children and adolescents with TS.

\section{Participants}

Study participants included children and adolescents aged five to seventeen years, with diagnosed TS and tics requiring treatment, according to the European treatment guidelines as described above [9]. Inclusion criteria furthermore included that patients should be willing to have a blood sample drawn before starting the treatment and again after three, six and 12 months. Exclusion criteria included children with fatty acid $\beta$-oxidation metabolic disorders, parents who were not able to read and/or understand oral and written information and children with other competing diagnoses.

\section{Screening and procedure}

Prospective participants and their families underwent a $1 \frac{1}{2}$-2-hour screening visit with clinical assistant (CBS) and/or the project nurse specialist (LA) after being informed about the project at a previous out-patient clinic visit with their responsible physician and showing interest in the project. This screening visit included 1) A presentation of the study and an example of diet plans; 2) Disease specific, demographic, medical and social history; 3 ) Data collection for body mass index

\section{Flowchart}

Modified Atkins Diet in treatment of severe tics

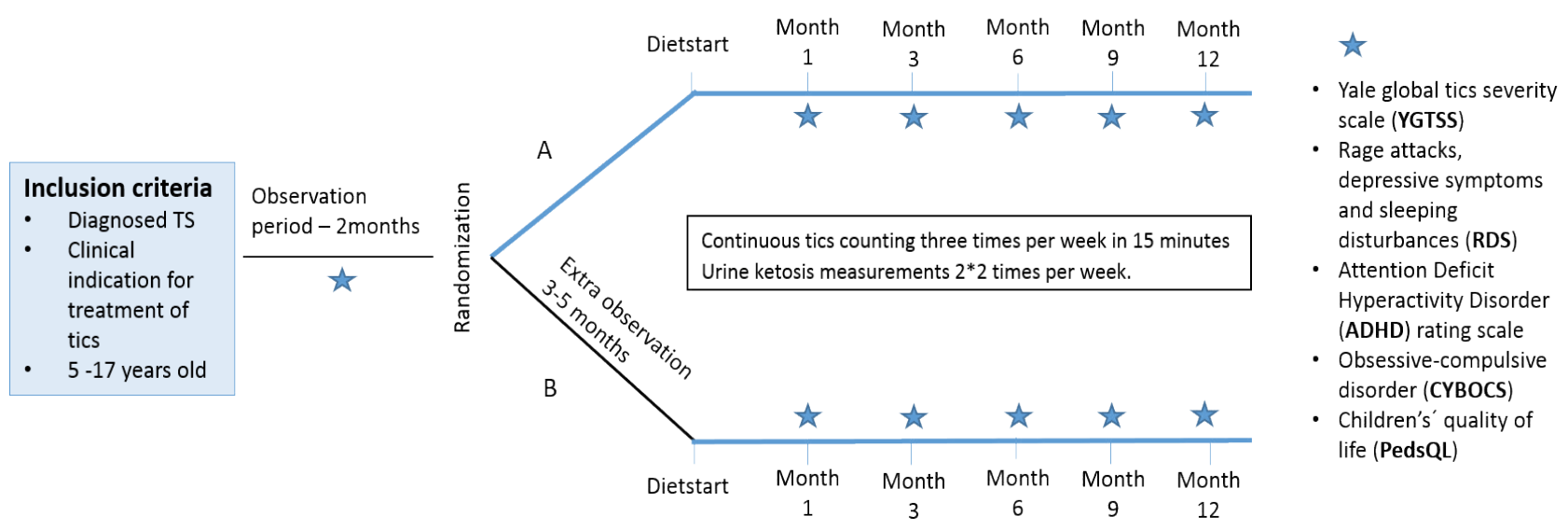

Figure 1: Study design of Modified Atkins Diet in treatment of severe tics.

TS = Tourette syndrome 
Citation: Sørensen CB, Skov L, Aaslet L, et al. (2021) Modified Atkins Diet for Tics Requiring Treatment in Tourette Syndrome: A Randomized Controlled Trial of Early Versus Late Initiation. J Pediatr Neurol Neurosci 5(1):86-94

(BMI); 4) Assessment of severity of tics with Yale Global Tic Severity Scale Score (YGTSS) [25]; 5) Information about blood samples that should be taken prior to starting the diet and after three, six and 12- months; 6) Handouts with comorbidity scales that should be handed in before next visit; 7) Information about tic-count at home by parents (three times per week in 15 minutes) and 8) written informed consent. CBS and LA assessed the first three patients together in order to minimize the inter rater variability.

Participants and their families were instructed to eat their usual diets in the baseline period until randomization and the diet-conversation with an experienced KD dietician (HN or $\mathrm{MM}$ ). After the two months baseline period participants were randomized to either start diet at this point in time (group A) or to an extra 3-5 months observation period before diet start (group B).

At diet start participants were seen by either CBS or LA to a pre-starting conversation with 1) Assessment of severity of tics with YGTSS, 2) Handouts with comorbidity scales, 3) Data collection for BMI, 4) Information about how to check for urine ketosis minimum twice per week (to ensure ketosis) and delivery of urine ketosis sticks. Furthermore, participants and their families had a diet- conversation (2-3 hours) with $\mathrm{HN}$ or MM who initiated the diet in an outpatient setting. All children still had tics that required treatment at time of diet start, mainly based on severity and impact of tics. The different treatment possibilities for tics were discussed with the families and if they were still interested in treatment with $M A D$ they were offered to be included in this study.

We offered a MAD starting on $10 \mathrm{~g}$ carbohydrates $(\mathrm{CH})$ per day, encouraging high fat and adequate protein [22]. $\mathrm{CH}$ could be increased after the first month on an individual basis up to $20 \mathrm{~g}$ per day. One researcher (CBS) was supporting the families throughout the whole period and families had the opportunity to contact this researcher daily. We had written diet material for the families, modified from what we use for families of children with epilepsy.

Hereafter, patients were seen and assessed by either LA or CBS after 1 month on the diet and every $3^{\text {rd }}$ month thereafter for one year (Figure 1). Diet compliance was determined by measuring urine ketones, which was done by the parents and they were instructed to contact CBs or LA if ketosis could not be achieved or fell.

\section{Outcome measurements}

We used validated diagnostic instruments to assess the severity of tics and the presence of comorbidities. YGTSS was used to assess the severity of tics [25]. The YGTSS is a clinician rated, multidimensional semi-structured scale. Multiple studies have shown good internal consistency, inter-rater reliability and validity in pediatric TS populations $[26,27]$.

Motor and vocal tics are rated separately and are each scored on a 0 to 5 scale across 5 dimensions; number, frequency, intensity, complexity, and interference caused in the last week. The scores from the 5 dimensions are summed and give the Total Motor Tic score (range 0-25) and the Total Vocal Tic score (range 0-25). By summing the Total Motor Tic score and the Total Vocal Tic score, Total Tic Severity score (range 0-50) is calculated. Patients are asked to rate the overall impairment (overall impact of tics, range 0-50) with 5 scoring anchors; 0 (none), 10 (minimal), 20 (mild), 30 (moderate), 40 (marked) and 50 (severe). Together the Total Tic Severity Score and the impairment score give the Total Yale Global Tic Severity Scale Score (range 0-100).

In this study we used the Total Motor Tic score, Total Vocal Tic score, Total Tic Severity score and Total Yale Global Tic Severity Scale Score.

Secondary outcome measures were effect on comorbidities and co-occurring symptoms; ADHD, OCD, rage attacks, sleeping disturbances, symptoms of depression and effect on quality of life. ADHD-Rating Scales (ADHD-RS) [28] and Children's Yale-Brown Obsessive Compulsive Scale (CY-BOCS) [29] were used to assess the presence of ADHD and OCD respectively. Quality of Life was examined by the Pediatric Quality of Life Inventory (PedsQL) [30].

\section{Statistics}

Descriptive statistics were used to assess age, sex, duration of diagnosis, duration of tics, baseline YGTSS scores, comorbidity scores, and quality of life scores.

A t-test was used to assess the effect of diet by comparing the different measurements times. To try to overcome the problem of missing data, a repeated measure model (GEE) was employed. In this model we assumed that for group $A$, children were dieting on measures 1 and 3 months but not dieting at baseline measurement. For group $B$, children were dieting at months 3 and 6 , but not dieting at baseline and start measurement.

\section{Results}

In total, 26 children were included. After an observation period of 2 months, children were randomized blindly into 2 groups, starting MAD immediately (group A) or after 3-6 months (group B). Tics were assessed after 1 month on the diet and every $3^{\text {rd }}$ month thereafter for 1 -year. Mean age at time of inclusion was 10.89 years (5.11-16.31, SD 2.82) and $69.2 \%$ were boys $(N=18)$ and $30.8 \%$ girls $(N=8)$.

The mean age at diagnosis was 7.90 years (4.0-13.0, SD 2, 19) and the mean age at onset of tics 5.04-years (2.0-8.0, SD 1.62). The mean duration of tics at inclusion was 5.93 years (1.41-13.31, SD 2.75), and the mean duration of diagnosis TS at inclusion was 2.99-years (0.41-6.74, SD 2.25).

6 children (24\%) received medical treatment at time of inclusion and 3 (12\%) had previously received medical treatment. One received treatment with Methylphenidate and Atomoxetin, 1 with Clonidine, one with Pimozide, one with Aripiprazole, one with Clonidine and Melatonin, and one with Seroquel.

As depicted in Figure 2, 24 children were randomized at baseline. Two patients dropped out before randomization one due to severe tics that needed acute treatment and one due to comorbid OCD. 13 Children (54.2\%) were randomized to group $A$ and 11 children (45.8\%) to group B. For the to- 


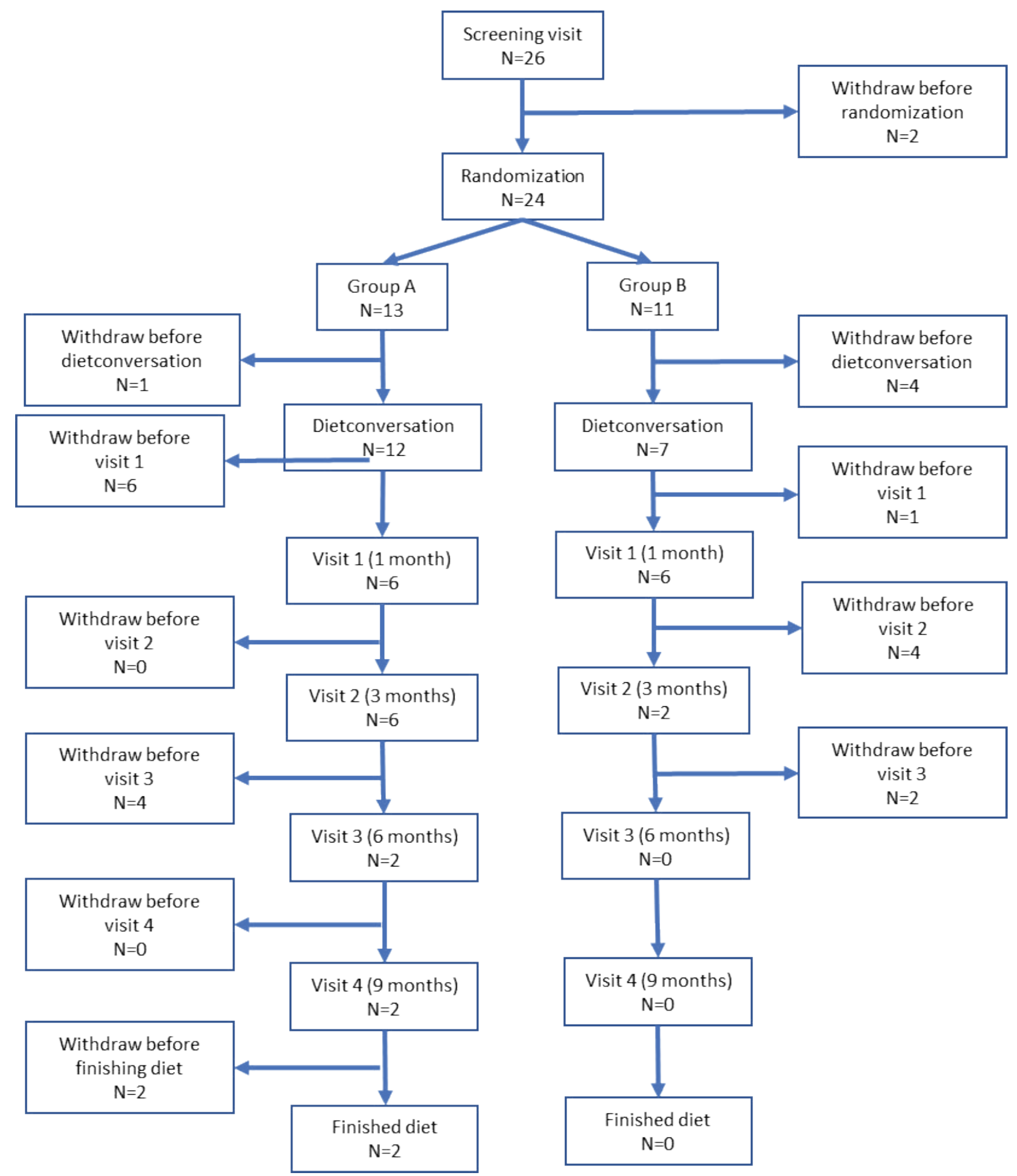

Figure 2: Flowchart of included participants.

tal cohort, the mean number of days in baseline period was 55.18 days (32-69, SD 8.72) and the mean number of days on diet was 65.62 days (0-343, SD 93.47).

Baseline characteristics of the total cohort, and the characteristics of the participants in group $A$ and $B$ respectively, are reported in Table 1. There were no statistically significant differences between the included participants in group $A$ and group B with regard to age at inclusion, baseline YGTSS scores, baseline comorbidity, baseline quality of life, duration of tics or number of days on diet (Table 1).

After one month on diet, $66.67 \%(\mathrm{~N}=8)$ reported side effects, after three months on diet $87.5 \%(\mathrm{~N}=7)$, after six months on diet $50 \%(\mathrm{~N}=1)$, and after 12 months none. The side effects were mild and the most commonly reported were 
Citation: Sørensen CB, Skov L, Aaslet L, et al. (2021) Modified Atkins Diet for Tics Requiring Treatment in Tourette Syndrome: A Randomized Controlled Trial of Early Versus Late Initiation. J Pediatr Neurol Neurosci 5(1):86-94

Table 1: Characteristics of included participants in the total cohort at baseline, and divided in group A (started diet immediately after randomization) and group B (started diet after an observation period of 3-5 months).

\begin{tabular}{|c|c|c|c|c|}
\hline & Total cohort $(\mathrm{N}=\mathbf{2 6})$ & Group A (N = 13) & Group B (N = 11) & p-value \\
\hline Age (SD) & $10.89(2.82)$ & $9.92(2.80)$ & $11.16(2.11)$ & $0.870^{1}$ \\
\hline Baseline Total Motor Tic Score (SD) & $15.0(3.71)(\mathrm{N}=25)$ & $14.62(4.25)(\mathrm{N}=13)$ & $15.80(3.33)(\mathrm{N}=10)$ & $0.502^{1}$ \\
\hline Baseline Total Vocal Tic Score (SD) & $9.52(5.37)(N=25)$ & $11.15(4.85)(\mathrm{N}=13)$ & $7.60(6.08)(\mathrm{N}=10)$ & $0.894^{1}$ \\
\hline Baseline Total Tic Severity Score (SD) & $24.6(7.47)(N=25)$ & $25.77(7.55)(\mathrm{N}=13)$ & $23.60(8.24)(\mathrm{N}=10)$ & $0.571^{1}$ \\
\hline Baseline Total Yale Global Tic Severity Scale Score (SD) & $40.2(13.55)(N=25)$ & $41.15(14.38)(N=13)$ & $41.60(12.97)(N=10)$ & $0.329^{1}$ \\
\hline Presence sleeping disturbance & $62.5 \%(N=16)$ & $77.8 \%(N=9)$ & $42.9 \%(N=7)$ & $0.274^{2}$ \\
\hline Presence rage attacks & $25.0 \%(N=16$ & $33.3 \%(\mathrm{~N}=9)$ & $14.3 \%(N=7)$ & $0.683^{2}$ \\
\hline Presence depressive symptoms & $18.8 \%(N=16)$ & $22.2 \%(\mathrm{~N}=9)$ & $14.3 \%(N=7)$ & $0.866^{2}$ \\
\hline Presence OCD & $37.5 \%(N=16)$ & $55.6 \%(\mathrm{~N}=9)$ & $14.3 \%(N=7)$ & $0.091^{2}$ \\
\hline Presence ADHD & $5.9 \%(N=17)$ & $0 \%(N=10)$ & $14.3 \%(N=7)$ & $0.218^{2}$ \\
\hline PedsQL Parents Psychosocial Health (SD) & $64.99(18.47)(\mathrm{N}=12)$ & $71.50(14.08)(N=5)$ & $60.33(20.81)(N=7)$ & $0.325^{1}$ \\
\hline PedsQL Parents Physical & $82.38(23.30)$ & $89.68(6.58)$ & $77.16(29.84)$ & \multirow[t]{2}{*}{$0.384^{1}$} \\
\hline Health (SD) & $(N=12)$ & $(N=5)$ & $(N=7)$ & \\
\hline PedsQL Parents Total score (SD) & $72.36(13.16)(N=12)$ & $77.82(8.13)(N=5)$ & $68.45(15.19)(\mathrm{N}=7)$ & $0.241^{1}$ \\
\hline Peds QL Child Psychosocial & $61.23(24.14)$ & $53.67(25.19)$ & $67.71(23.03)$ & \multirow[t]{2}{*}{$0.316^{1}$} \\
\hline Health (SD) & $(N=13)$ & $(N=6)$ & $(N=7)$ & \\
\hline PedsQL Child Physical Health (SD) & $78.18(27.09)(\mathrm{N}=13)$ & $67.75(37.19)(\mathrm{N}=6)$ & $87.13(10.62)(\mathrm{N}=7)$ & $0.212^{1}$ \\
\hline PedsQL Child Total score (SD) & $67.72(23.40)(\mathrm{N}=13)$ & $59.63(28.04)(N=6)$ & $74.66(17.86)(\mathrm{N}=7)$ & $0.266^{1}$ \\
\hline Duration of tics (in years) & $5.93(2.75)$ & $5.31(2.43)$ & $5.59(1.92)$ & $0.647^{1}$ \\
\hline Number of days on diet & $65.62(93.47)$ & 91.69 (119.54) & $46.73(50.58)$ & $0.729^{1}$ \\
\hline
\end{tabular}

SD = Standard Deviation; PedsQL = Pediatric Quality of Life Inventory; 1 = One-Way Anova; 2 = Pearson Chi-Square

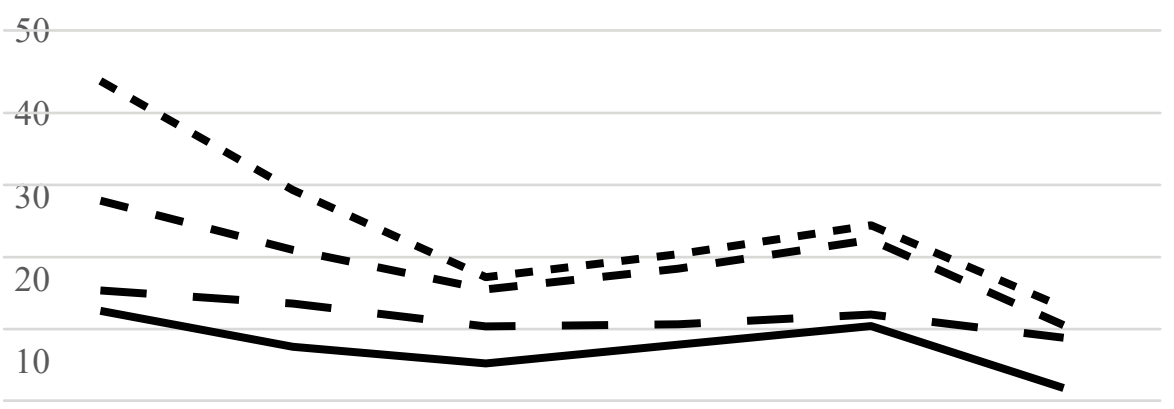

0

Baseline One month Three months Six months Nine months Twelve months

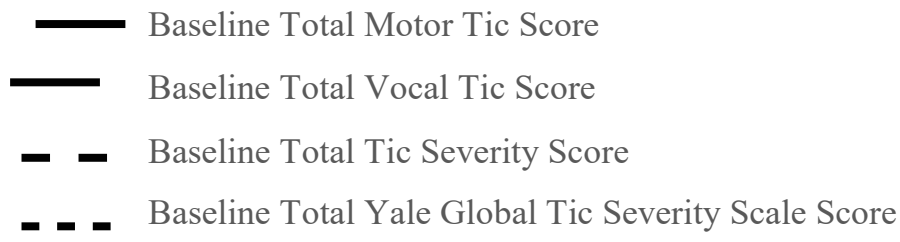

Figure 3: Tic scores of the participants $(\mathrm{N}=6)$ who have been on diet for 100 days or more.

tiredness, nausea, constipation, enuresis nocturna and nosebleeds.

In total, 6 children were on diet for 100 days or more. The characteristics of these 6 children are described in Figure
3. The mean age of these six children was 8.04 years (range $5.11-11.91, \mathrm{SD} 2.75)$ and $66.7 \%$ was male $(\mathrm{N}=4)$ and $33.3 \%$ female $(\mathrm{N}=2)$. 
Citation: Sørensen CB, Skov L, Aaslet L, et al. (2021) Modified Atkins Diet for Tics Requiring Treatment in Tourette Syndrome: A Randomized Controlled Trial of Early Versus Late Initiation. J Pediatr Neurol Neurosci 5(1):86-94

The effect of diet was assessed by comparing different measurements times in the two groups. Group A was on diet from baseline to the measurement three months while group $B$ was on diet from month 3 and 3 months onwards. The results can be seen in Table 2. There were no significant differences between the groups or over time.

Any conclusions about tendencies in the data would be untrustworthily due to the high missing fractions.

To try to overcome the problem of missing data, a repeated measure model (GEE) was employed. This model yielded a tendency towards a positive effect of diet $(p=0.1025)$ between treatment groups. The effect estimate is -0.6423 indicating that the diet group is around 0.5 unit lower in YGTSS on average.

\section{Discussion}

The aim of our study was to investigate whether a MAD could be beneficial for children and adolescents with tics requiring treatment, in reducing tics frequency and severity with fewer adverse effects than pharmacological therapy. We used a randomised approach with 2 groups of children with TS who started KD at different time points.

We found a reduction in tics severity and frequency in few children who stayed on the diet up to 1-year. However, we could not show a significant statistical difference between the groups, but a trend towards positive diet effect $(p=0.1025)$. The effect estimate of the diet was found to be -0.6423 indicating that the diet group is around 0.5 unit lower in YGTSS on average, which is a very little effect on the total YGTSS score.

The original design turned out to be difficult to complete according to protocol. There was planned a YGTSS score at baseline and at diet start, but some families did not want to come to the hospital in order to be YGTSS scored and were started on the diet at home after a visit of the dietician.

Furthermore, the parents were asked to count tics at home once a week to monitor the fluctuation of tics, but it was only performed by a few families, and therefore these measures could not be included in the statistical analyses. Many children were not able to complete the study, and this is probably one of the main reasons for not being able to conclude whether the MAD influences tic severity in patients with TS or not.

The high dropout rate could probably be explained by several reasons. First, radically dietary changes are difficult in all ages, but are probably better maintained in very young children with for example intractable epilepsy, where parents are highly motivated to carry out the treatment in the hope of eliminating harmful seizures and improving quality of life [31]. For most of the participants in our study, difficulties in keeping the diet seemed to be a bigger hurdle, higher than the difficulties experienced by having the disease and even being highly motivated before inclusion.

Parents reported that the diet was very time-consuming, expensive, that their children didn't like the food and that the social impediments were too big compared to the positive treatment effect.

Participants often reported that they missed being like the other children in school, being able to eat the same kind of food in lunch breaks and at birthday parties.

Secondly, we didn't choose a cut-off value on tics severity to enter the study. Perhaps participants with higher impairment and Total Yale Global Tic Severity Scale Score on the YGTSS would have been more motivated to carry out the treatment. For example, one of the patients who remained on diet for 343 days had Total Global Yale Tic Severity Scale score of 54 at baseline, 34 after one month on diet, 9 after three months on diet, 9 after six months on diet and 15 after 9 months.

Regarding adverse effects, none of the children or adolescents experienced severe adverse effects and the diet was generally well tolerated. With regard to reported adverse effects we cannot confirm or deny a causal link, but we have registered the prevalence of accompanying symptoms.

Participants were followed with blood samples every three months; in none of the cases we found changes that required termination of the diet.

A previous study using a MAD for treating headaches [32] experienced similar limitations because of difficulties in maintaining the diet. However, some participants experi-

Table 2: Summary of t-test results of assessment of effect of diet.

\begin{tabular}{|l|l|l|l|l|l|}
\hline & Estimate & $\begin{array}{l}\text { Lower } 95 \text { pct } \\
\text { Cl }\end{array}$ & $\begin{array}{l}\text { Upper } 95 \text { pct } \\
\text { Cl }\end{array}$ & p-value & $\begin{array}{l}\text { Number of } \\
\text { valid data }\end{array}$ \\
\hline Change in group A & -1.4 & -4.12 & 1.32 & 0.226 & 5 \\
\hline Change in group B & NA & NA & NA & NA & 1 \\
\hline Group difference in Change & NA & NA & NA & 6 \\
\hline BL (A vs. B) & -0.262 & -1.213 & 0.689 & 0.574 & 23 \\
\hline 3M (A vs. B) & -2.3 & -5.233 & 0.633 & 0.099 & 7 \\
\hline 6M (A vs. B) & 1 & -2.284 & 4.284 & 0.495 & 9 \\
\hline BL in group A vs. 3M in group B & -0.962 & -2.767 & 0.844 & 0.271 & 15 \\
\hline 3M in group A vs. 6M in group B & 0.2 & -4.311 & 4.711 & 0.908 & 6 \\
\hline
\end{tabular}

$\mathrm{Cl}=$ Confidence Interval; $\mathrm{BL}=$ baseline; $3 \mathrm{M}=$ Measurement at 3 months, $6 \mathrm{M}=$ Measurement at 6 months 
enced some positive effects and continued the diet. In our study we have experiences with a few children who stayed on the diet for up to 1-year. Whether an initial effect of the diet on tics was the main motivating factor for continuing in the study or whether maintaining the diet enough time resulted in a positive effect we cannot answer. However, there were insufficient study completers to analyze statistically for any potential beneficial aspects of those who seemed to respond.

Previous studies have examined the effect of complementary therapies in patients with TS such as omega-3 fatty acids supplementation (O3FA) [33,34] and dietary changes with gluten free diets [35]. Clinical observations have suggested an effect of O3FA in patients with TS, but a placebo controlled trial with 33 children and adolescents was not able to show a reduction in tic scores [34]. A prospective pilot study looked at the effect of a gluten free diet and found that children and adults who maintained the diet for one year had a marked reduction in tics and OCD [35].

Secondary outcome measures were effect on comorbidities. Due to missing data and too few study completers, there were not enough data to analyze statistically for potential beneficial aspects on comorbidities. One of the reported comorbidities in our study participants was ADHD. A previous prospective study of children with epilepsy reported that after one year on the KD, there was a statistically significant improvement of both attention and social functioning [36]. Whether this is related to the reduction of seizures or it might be an independent effect, we don't know. However, some epilepsy studies have reported positive effect of the KD on sleep, behaviour, and alertness among others, even in the lack of effect on seizures [37-39]. However, to our knowledge, there are not specific studies on ADHD and KD that show a statistically significant effect of the KD on ADHD [40]. Evidence regarding $A D H D$ and $K D$ in humans is lacking, but a randomized, placebo- controlled study compared the effects of KD and a standard diet on behavior in 21 dogs with epilepsy and comorbid ADHD [41]. When the KD was compared with the standard diet, it showed a significant improvement in ADHD-related behaviors.

Study strengths were that all the children were scored by 2 experienced clinicians in TS (CBS, LA) who evaluated the children in a very systematic way. Likewise, the 2 dieticians were dedicated KD dieticians with many years of experience with KD in epilepsy. That meant very close and dedicated support; new recipes individually adapted to the children's preferences and frequent counselling were available throughout the whole study period.

Certain study weaknesses should be mentioned. First, as mentioned before, there were many withdrawals. Second, there were many missing data primarily due to missing data delivery from participants, especially comorbidity assessment scales. This could probably have been improved if these assessment tools were filled out in the clinic during the scheduled appointment.

We indirectly monitored patient adherence by training participants and/or families to self-monitor urine ketone levels with urine dipsticks at home. We chose urine dipstick tests over blood ketone levels due to the age of the participants and for the sake of simplicity. We did not get these results from the participants, which could have helped us determine whether missing ketosis could explain for example lack of effect, though our impression is that parents contacted CBS if ketone levels where low to ensure guidance in restoring ketosis.

KDs will, in the near future, hopefully become a part of our non-pharmacological treatment options at the Tourette clinic at the department of Paediatrics, Herlev University Hospital. The MAD could be a "rescue choice" for short periods of severe tics, instead of medicines. The treatment might be repeated more than once throughout infancy, if needed in periods with severe tics. The side effects were few and mild. In our experience, a few weeks are sufficient for showing potentially beneficial effects.

\section{Conclusion}

In conclusion, the diet was well tolerated but difficult to maintain for many children, even experiencing reduction in tics. We found a reduction in tics severity and frequency in few children who stayed on the diet for up to 1-year. However, we could not show a significant statistical difference between the groups, but a trend towards positive diet effect ( $p$ $=0.1025)$.

This is, to our knowledge, the first study where a KD is tried in a randomized prospective way in children with TS. As the compliance was low, this treatment option is probably not preferable for the majority of patients with TS, but might be an option in selected cases, also depending on the parental motivation. In a future study, we suggest that patients with affected quality of life or severe tics will be included in order to increase the compliance of the families and the homogeneity of the included patients. Randomized trials with adequately powered sample size and control group are needed in the future before KD might be offered as part of routine practice. Our goal is to offer MAD as rescue therapy in short periods for selected children with severe tics and maybe perform a larger study. Research materials can be accessed by contacting the corresponding author.

\section{Disclosure Section}

\section{Acknowledgment}

The work was done at the department of paediatrics, Herlev University Hospital, Denmark. Concept of the study was presented at Global Symposium for Ketogenic Dietary Therapies, England, 2014, at Danish Neuropediatric Society Meeting, Denmark, 2014, and at Nordic Ketogenic Diet meeting, Denmark, 2015. Preliminary results were presented at $6^{\text {th }}$ Global Symposium on Ketogenic Therapies for Neurological Disorders, Korea, 2018.

\section{Author contribution}

Sørensen C contributed to conception and design, contributed to acquisition, analysis and interpretation, drafted the manuscript, critically revised manuscript and gave final approval. Skov L contributed to conception and design, con- 
Citation: Sørensen CB, Skov L, Aaslet L, et al. (2021) Modified Atkins Diet for Tics Requiring Treatment in Tourette Syndrome: A Randomized Controlled Trial of Early Versus Late Initiation. J Pediatr Neurol Neurosci 5(1):86-94

tributed to interpretation, critically revised manuscript and gave final approval. Aaslet $L$ contributed to acquisition, critically revised manuscript and gave final approval. Nielsen $\mathrm{H}$ contributed to acquisition, critically revised manuscript and gave final approval. Mortensen $\mathrm{M}$ contributed to acquisition, critically revised manuscript and gave final approval. Lange $T$ contributed to analysis and interpretation, drafted the manuscript, critically revised manuscript and gave final approval. Debes $\mathrm{N}$ contributed to conception and design, contributed to analysis and interpretation, drafted the manuscript, critically revised manuscript and gave final approval. Miranda $M$ contributed to conception and design, contributed to analysis and interpretation, drafted the manuscript, critically revised manuscript and gave final approval. Debes $\mathrm{N}$ and Miranda $\mathrm{M}$ are mentors who contributed equally to this work.

\section{Declaration of Conflicting Interests}

The Authors declare that there is no conflict of interest.

\section{Funding}

The authors disclosed receipt of the following financial support for the research, authorship, and/or publication of this article: This work was supported by Glashofs Legat and Dronning Louise Børnehospitals Fond.

\section{Ethical Approval}

The study was approved by The Scientific Ethical Committees (protocol H-1-2013-095) and the Danish Data Protection Agency (protocol IS03531/HEH-2015-015). We obtained informed signed consent in all cases.

\section{References}

1. Association AP Diagnostic and Statistical Manual of Mental Disorders (DSM-5). ( $5^{\text {th }}$ edn), American Psychiatric Publishing, Washington, DC, USA

2. Bloch MH, Leckman JF (2009) Clinical course of Tourette syndrome. J Psychosom Res 67: 497-501.

3. Freeman RD, Fast DK, Burd L, et al. (2007) An international perspective on Tourette syndrome: Selected findings from 3500 individuals in 22 countries. Dev Med Child Neurol 42: 436-447.

4. Groth C, Mol Debes N, Rask CU, et al. (2017) Course of Tourette Syndrome and Comorbidities in a Large Prospective Clinical Study. Journal of the American Academy of Child and Adolescent Psychiatry 56: 304-312.

5. Mol Debes NMM (2013) Co-morbid disorders in Tourette syndrome. Behav Neurol 27: 7-14.

6. Mol Debes NMM, Skov L, Hjalgrim H (2008) Tourette syndrome. Genetics, neuroanatomy and neurotransmitters. Ugeskr Laeger 170: 2695-2700.

7. Augustine F, Singer HS (2019) Merging the pathophysiology and pharmacotherapy of tics. Tremor and Other Hyperkinetic Movements 8: 595.

8. Scahill L, Erenberg G, Berlin CM, et al. (2006) Contemporary Assessment and Pharmacotherapy of Tourette Syndrome. NeuroRx 3: 192-206.

9. Roessner V, Plessen KJ, Rothenberger A, et al. (2011) European clinical guidelines for Tourette syndrome and other tic disorders.
Part II: Pharmacological treatment. Eur Child Adolesc Psychiatry 20: 173-196.

10. Verdellen C, van de Griendt J, Hartmann A, et al. (2011) European clinical guidelines for Tourette syndrome and other tic disorders. Part III: Behavioural and psychosocial interventions. Eur Child Adolesc Psychiatry 20: 197-207.

11. Azrin NHH, Nunn RGG (1973) Habit-reversal: A method of eliminating nervous habits and tics. Behav Res Ther 11: 619-628.

12. McGuire JF, Ricketts EJ, Piacentini J, et al. (2015) Behavior Therapy for Tic Disorders: an Evidenced-Based Review and New Directions for Treatment Research. Curr Dev Disord Reports 2: 309-317.

13. Joseph F, McGuire JP, Erin A. et al. (2014) A meta-analysis of behavior therapy for Tourette Syndrome. J Psychiatr Res 50: 106112.

14. van de Griendt JMTM, van Dijk MK, Verdellen CWJ, et al. (2018) The effect of shorter exposure versus prolonged exposure on treatment outcome in Tourette syndrome and chronic tic disorders - an open trial. Int J Psychiatry Clin Pract 22: 262-267.

15. Hoogduin K, Verdellen C, Cath D (1997) Exposure and response prevention in the treatment of gilles de la Tourette's Syndrome: Four Case Studies. Clin Psychol Psychother 4: 125-135.

16. Steeves T, McKinlay BD, Gorman D, et al. (2012) Canadian guidelines for the evidence-based treatment of tic disorders: Behavioural therapy, deep brain stimulation and transcranial magnetic stimulation. Can J Psychiatry 57: 144-151.

17. Kossoff EH, Zupec-Kania BA, Auvin S, et al. (2018) Optimal clinical management of children receiving dietary therapies for epilepsy: Updated recommendations of the International Ketogenic Diet Study Group. Epilepsia Open 3: 175-192.

18. Barañano KW, Hartman AL (2008) The ketogenic diet: Uses in epilepsy and other neurologic illnesses. Curr Treat Options Neurol 10: 410-419.

19. Stafstrom CE, Rho JM (2012) The ketogenic diet as a treatment paradigm for diverse neurological disorders. Front Pharmacol 3: 59.

20. Masino SA, Rho JM (2019) Metabolism and epilepsy: Ketogenic diets as a homeostatic link. Brain Res 1703: 26-30.

21. Rogawski MA, Löscher W, Rho JM (2016) Mechanisms of action of Antiseizure Drugs and the Ketogenic diet. Cold Spring Harb Perspect Med 6: a022780.

22. Rho JM (2017) How does the ketogenic diet induce anti-seizure effects? Neurosci Lett 637: 4-10.

23. Baj A, Moro E, Bistoletti M, et al. (2019) Glutamatergic signaling along the microbiota-gut-brain axis. Int J Mol Sci 20: 1482.

24. Dahlin M, Mansson JE, Amark P (2012) CSF levels of dopamine and serotonin, but not norepinephrine, metabolites are influenced by the ketogenic diet in children with epilepsy. Epilepsy Res 99: 132-138.

25. Leckman JF, Riddle MA, Hardin MT, et al. (1989) The yale global tic severity scale: Initial testing of a clinician-rated scale of tic severity. J Am Acad Child Adolesc Psychiatry 28: 566-573.

26. Storch EA, Murphy TK, Geffken GR, et al. (2005) Reliability and validity of the Yale Global Tic Severity Scale. Psychol Assess 17: 486-491.

27. McGuire JF, Piacentini J, Storch EA, et al. (2018) A multicenter 
examination and strategic revisions of the yale global tic severity scale. Neurology 90: e1711-e1719.

28. Szomlaiski N, Dyrborg J, Rasmussen H, et al. (2009) Validity and clinical feasibility of the ADHD rating scale (ADHD-RS) A Danish Nationwide Multicenter Study. Acta Paediatr 98: 397-402.

29. Scahill L, Riddle MA, Mcswiggin-Hardin M, et al. (1997) Children's Yale-Brown Obsessive Compulsive Scale: Reliability and Validity. J Am Acad Child Adolesc Psychiatry 36: 844-852.

30. Varni JW, Seid M, Kurtin PS (2001) PedsQL 4.0: Reliability and validity of the Pediatric Quality of Life Inventory version 4.0 generic core scales in healthy and patient populations. Med Care 39: 800-812.

31. Winesett SP, Bessone SK, Kossoff EHW (2015) The ketogenic diet in pharmacoresistant childhood epilepsy. Expert Rev Neurother 15: 621-628.

32. Kossoff EH, Huffman J, Turner Z, et al. (2010) Use of the modified Atkins diet for adolescents with chronic daily headache. Cephalalgia 30: 1014-1016.

33. Quezada J, Coffman KA (2018) Current approaches and new developments in the pharmacological management of tourette syndrome. CNS Drugs 32: 33-45.
34. Gabbay V, Babb JS, Klein RG, et al. (2012) A double-blind, placebo-controlled trial of omega-3 fatty acids in Tourette's disorder. Pediatrics 129: 1493-1500

35. Rodrigo L, Álvarez N, Fernández-Bustillo E, et al. (2018) Efficacy of a gluten-free diet in the gilles de la tourette syndrome: A pilot study. Nutrients 10: 573.

36. Pulsifer MB, Gordon JM, Brandt J, et al. (2007) Effects of ketogenic diet on development and behavior: Preliminary report of a prospective study. Dev Med Child Neurol 43: 301-306.

37. Van Berkel AA, IJff DM, Verkuyl JM (2018) Cognitive benefits of the ketogenic diet in patients with epilepsy: A systematic overview. Epilepsy Behav 87: 69-77.

38. Hallbook T, Kohler S, Rosen I, et al. (2007) Effects of ketogenic diet on epileptiform activity in children with therapy resistant epilepsy. Epilepsy Res 77: 134-140.

39. Hallbook T, Lundgren J, Rosen I (2007) Ketogenic Diet Improves Sleep Quality in Children with Therapy-resistant Epilepsy. Epilepsia 48: 59-65.

40. Bostock ECS, Kirkby KC, Taylor BVM (2017) The current status of the ketogenic diet in psychiatry. Front Psychiatry 8: 43.

41. Packer RMA, Law TH, Davies E, et al. (2016) Effects of a ketogenic diet on ADHD-like behavior in dogs with idiopathic epilepsy. Epilepsy Behav 55: 62-68. 